\title{
Reading Nonlinear Digital Narratives: Viewing Strategies of 21st Century Learners
}

\author{
Neliza C. Casela \\ Adamson University, Manila, Philippines
}

\begin{abstract}
This phenomenological study avers that mental processes in critical viewing and reading are similar. It determines the learners' literal and critical processing strategies in viewing nonlinear digital narratives (NLDNs), like science fiction and epic fantasy films, similar to strategies in reading science fiction and epic fantasy texts; and explores their affective and interactive responses while viewing which relate to affective factor in reading. Viewers, like readers, use literal and critical processing strategies in viewing films by using their narrative and cultural schemata and by mapping out narrative details. Mapping their schemata with the film makes them find and discover new strategies, combining them to proceed with their critical viewing and processing. Viewers creatively use and generate language to represent concepts, explain ideas, and express emotions. They also use punctuation marks or emoticons to convey emotions, reactions, or thoughts. Their appropriate affective and interactive responses are associated to their analytical processing of scenes or events. Likewise, readers manifest, demonstrate, or express affective and interactive while processing print.
\end{abstract}

Keywords: critical reading, critical viewing, mental or cognitive processing, processing strategies, affective and interactive responses

\section{Introduction}

In determining the rising literacy demands of the 21st century, Christie (1990, as cited in McKay, 1993) described a more complex phenomenon and more sophisticated literacy such as new kinds of reading, writing or viewing for processing new genres from digital media or cyber technology, and generating new knowledge. The narrative schema, processing strategies, and engagement of learners in interactive fiction may influence their reading and viewing skills.

Significantly, media literacy recognizes the comparison between media forms and print texts considered as forms of composition which seem to require certain skills, and result to meaning making. Media forms can be similarly read, since the signs can be decoded, the texts analyzed, the forms of representation understood, engaged in, enjoyed in ways analogous to the reading of print in general, and literature more specifically.

Dancyger (2011) pointed out that digital technology will yield new forms of storytelling, new levels of interactivity, and a more democratic relationship between storyteller (filmmaker) and audience. Heston, McGovern, and Cameron ( as cited in Ohanian \& Phillips, 1996) all acknowledged the value of emerging interactive films.

Neliza C. Casela, professor, Ph.D., Center for Research, Evaluaton, and Continuing Education (CRECE), Adamson University. 
This study holds that like reading, viewing is an active, constructive, and interactive activity, which involves processing, and affective and interactive responses. Processing strategies of learners in viewing films are almost similar to reading print. Moreover, affective and interactive responses in viewing film are also cognitive because processing the film codes influences and draws appropriate responses, much like in reading.

Reading and viewing are incidentally visual perception, but both require the learner to process information. The central premise involves the concept of schema (Bartlett, 1932; as cited in Carbon \& Albrecht, 2012; Rumelhart, 1980; as cited in Mandler, 1984), which recognizes the role of reader's schema, like the viewer's schema, to comprehend and learn from the text. The reader, like the viewer, comprehends by using his existing knowledge, but which can change when he encounters new information. Change indicates the reader's or the viewer's new learning integrated in his/her existing knowledge so that with this notion, the reader, like the viewer, uses the new information to modify his/her learning. Another theorician, Mandler (1984) argued that in constructing a story while following it, and in reconstructing it in memory, a reader or a viewer works with gist - the essential events and points. She recognized that top-down processing is likely to be extracting structural essentials from the text according to familiar patterns.

The interactive nature of reading and the reader's role in constructing meaning practically apply to viewing. Stanovich (1980) emphasized that meaning is created through the interaction of text and reader. In viewing, the interaction happens between the viewer and film considered as text to be processed, responded to, and interacted with.

Piaget's (1952) theory of disequilibrium postulates strong relationship between cognition and emotions has greatly influenced investigations sharing similar arguments that resistance or attempts to assimilation of new information into schema are associated with affects and emotions. Rosenblatt (1991) described that aesthetic experience in literature involves reading with attention, but mainly to what readers are experiencing, thinking, and feeling during reading. Viewing films, much like reading literature, brings a viewer aesthetic experience (Rosenblatt, 1991). Viewing films fulfills the viewers' aesthetics by expressing their emotions as they introspect and generate judgments on issues and conflicts.

The study claimed the following: viewing film, perceiving film codes, and responding to the film (affective and interactive) involve cognition, thus may be considered cognitive processing; viewing film, like reading, involves complex mental activity via the simultaneous interplay of processing the digitized visual and audio codes, and interacting in the virtual world of digital narrative; nonlinear digital narratives (NLDNs), like narrative in print, activates viewer's cognitive processing, and elicits active interaction of the viewer, the film, and the filmmaker; NLDNs involve the viewer in unconventional causal development of events which may demand more advanced viewing, processing, comprehension, and thinking; the viewer, like the reader, uses various strategies in processing the subtitle along with the dialogue and sound effects; and the viewer, like the reader, actively constructs and completes the meaning of film. The reader, like the viewer, uses many different areas of knowledge, ranging from print features, facts, and to strategies. In this study, the viewer interacts with films, likewise the reader interacts with texts.

In this study, science fiction and epic fantasy films are rendered in NLDNs, like narrative texts in print, defy linear plot but may activate viewers' nonlinear thinking and processing, and compel or require them to employ more critical analysis and interpretation. This nonlinearity may challenge the viewers to employ available strategies and find other strategies to resolve any deviation or unconventionality in the narrative structure, visual 
codes or virtual reality. This nonlinearity leads to the simultaneous or concurrent interplay of processing, interactive and affective responses. Nonlinearity may compel the viewers to actively process, participate, interact, respond, or even take a role as character in the narrative or inside the film environment, likewise a reader interacts with the context of a narrative.

\section{Statement of Purpose}

This study attempted to determine the processing strategies employed by the learners when viewing NLDNs, as input to developing a visual literacy model for 21st century learners.

Specifically, this study sought to: (1) explore the recurring information provided by the viewers to demonstrate their processing strategies and (2) explore the viewers' affective and interactive responses that relate to their cognitive processing strategies in viewing films.

\section{Methods}

This phenomenological study conducted concurrent viewing and transcribing thoughts to elicit the students' processing of science fiction and epic fantasy films. Concurrent reporting (Ericsson and Simon, 1993, as cited in van Gog and Paas, 2008), or thinking aloud is verbal reporting for thought processes and cognitive actions. It requires students to verbalize all thoughts that come to mind during task performance. This study involved actual concurrent viewing and transcription of thoughts analyzed by open, axial, and selective codings (Strauss \& Corbin, 1990; 1998, as cited in Leedy \& Ormrod, 2001).

In this study, the Viewer-Transcription Of Thought Protocol (VTTP) is based on the concept of the think-aloud protocol (TAP) in reading. The thought protocols are transcribed and subjected to analysis that led to development of a visual literacy model depicting the complex processes involved in the actual viewing, describing how these processes led to the participants' critical analysis and interpretation of the NLDNs, and explaining how these processes establish that science fiction and epic fantasy films are agents or media that activate the simultaneous processing of the interplay of visual and audio codes during the actual viewing, and establish active interaction of the viewer to the films.

During the actual viewing, viewers were prompted to note down details from the NLDN that they considered striking because the details are important, appealing, interesting, intriguing, questionable, or unbelievable. Concurrently, they wrote down their thoughts, reactions, feelings, or comments to every detail they noted. Their protocol sheet was divided into two: first column-details from the NLDNs; second column-VTTP. The data obtained from the first column indicate that the viewers found, decoded, and interpreted the intrinsic meaning of the NLDNs that accounts for the viewers' literal processing strategies. Moreover, the data obtained from the VTTP indicate that viewers actively construct the meaning of the NLDN which accounts for the viewers' critical processing strategies. The VTTP also revealed viewers' affective and interactive responses shown in their use of verbal tools and symbols.

The NLDNs viewed were based on famous science fiction The War of the Worlds (1898) (with original novel version by Herbert George Wells and 3D film version by Steven Spielberg), and epic fantasy The Lord of The Rings: The Return of the King (1955) (with original trilogy novel version by John Ronald Tolkien and 3D film version by Peter Jackson). 


\section{Results}

Literal processing strategies refer to strategies in viewing films when viewers use their narrative schema - the schema on narrative elements which include dialogue, setting, plot, character which were recurrently processed by the viewers. Critical processing strategies refer to strategies in viewing films when viewers employ their schemata: cultural schema, real world schema or verisimilitude. Both literal and critical processing involve pattern matching or schema fitting when they discriminate any mismatch. In the same manner, in reading science fiction and epic fantasy novels, readers process the narrative elements by employing both literal and critical reading strategies.

Viewers like readers process narrative details simultaneously/concurrently. Viewers' like readers' critical processing of narrative structure allows them to synthesize the narrative details, to coherently weave or put the details into a holistic narrative, and to complete the meaning of the film or the text. Synthesis involves analysis, confirmation or rejection of inference, prediction, and judgment.

Viewers like readers analyze events and actions in relation to character details/characterization. Processing allows them to synthesize the meaning of the film through the theme. They discriminate between positive and negative action/situations that made them experience positive or negative emotions, but made them judge apt to their cultural schemata. They employ suspension of disbelief in processing plot both in film and print. However, viewers have distinct strategy for processing virtual reality through the visual and sound effects, which readers do not possess since the element of virtual reality is only present in NLDNs. Readers process the mimetic depiction of the world only by the narrative description and progression provided in the text.

Verbal tools refer to the viewers' use of various strategies in communicating their thoughts, emotions, or reactions by utilizing, creating, generating words or expressions while processing the film. Verbal tools further show the viewers' creative use and generation of language to represent concepts, explain ideas, and express emotions. They process the visual codes of the film by assigning words or expressions, or by labeling. Moreover, processing through symbols refers to the use of punctuation marks or emoticons to convey emotions, reactions, thoughts, or meaning based on intensity or frequency/recurrence. Marginal notes while reading indicate readers' processing and interaction with the text through verbal tools and symbols.

Paivio' s dual-code theory of information processing (1971, as cited in Seel, 2008) guides the processing of virtual reality via visual and audio codes rendered in setting, plot, or characterization. The dual codes of NLDNs applied in scenes, actions, setting, computer-generated characters, creatures, or images accompanied by narration, and even subtitle are simultaneously processed by the viewers. Paivio's dual-code theory of information processing (1971, as cited in Seel, 2008), Lohr and Gall (2008), Levie and Lentz (1982, as cited in Seel, 2008), and R. E. Mayer (2001; 2002, as cited in Seel, 2008) suggest that information across two channels, the processing of nonverbal objects by means of imagery, and language can serve as reciprocal reinforcement and enhance both recall and comprehension. R. E. Mayer and Gallini (1990, as cited in Seel, 2008), revealed that visual cues amplify and explain text and facilitate the recall of new knowledge because visual cues create imagery during learning that is critical to memory processes. The audio-video redundancy of Seel (2008) attempts to explain how people integrate and learn information presented verbally and visually. Metz (1974; 1982 as cited in Mast, Cohen, \& Braudy, 1992) explained that each image freely created and arranged into an 
intelligible sequence, brings about the essence of semiological dimension of film. Virtual reality has increased images in filmmaking that every image can be possibly created and every new image can be generated which become accurate representation of real images or objects, likewise sounds. Processing these codes makes the viewers accept, reject, or question the mimetic interpretation of reality, or suspend disbelief. The dual code applies to reading such that visual pertains to print and verbal pertains to the language of the text. Reading is language in print, thus readers construct meaning by processing the print and language.

Processing information while viewing, several affective states potentially occur, like in reading. Smith (1995, as cited in Rushton \& Bettison, 2010) pertains to emotional simulation-imagining from the inside the affective states of characters, and explains the affective responses of viewers. Processing film details through affective responses is conveyed in the verbal tools and symbols appropriate to the setting and scenes rendered with visual and sound effects. The verbal tools convey audible sound based on the choice of words, tone, made evident with the use of enclitics and symbols such as punctuation marks, and emoticons suggesting strong emotions that vary in terms of repetition, capitalization indicating affect bursts such as fear, amazement, sighs, laughter, cries which Plutchik and Conte (1997) referred to as intensity or arousal. Axelrod and Hone (2006) recognized that people feeling the same thing might use different words in description, and using the same words might be describing different experiences. To reiterate, marginal notes of readers may account for their processing of text through verbal tools and symbols which may reveal their affect while reading.

J. L. Singer and D. G. Singer (1983, as cited in Seel, 2008), particularly investigated negative emotions that relate to inadequate schemata for processing information or cognitive disequilibrium which has high likelihood for activating conscious and effortful cognitive deliberations, questions, and inquiry that aim to restore cognitive equilibrium. Viewers' like readers' sadness, dismay, anger are caused by the scenes or events that contradict their expectations. Rozin and Cohen (2003, as cited in Seel, 2008) and Craig, Driscoll, and Gholson (2004, as cited in Seel, 2008), recognized confusion as an important affective state for learning. Uncertainty, confusion, or difficulty indicates cognitive disequilibrium due to inadequate schemata when viewers are not particularly interested in or have not viewed or read science fiction or epic fantasy. Seel (2008) claimed that cognitive disequilibrium has a high likelihood for activating conscious and effortful cognitive deliberations, questions, and inquiry that aim to restore cognitive equilibrium. Viewers like readers raise questions or inquiries, predict or infer when scenes or events seem not to match their expectations because of the nonlinear plot. Analepsis, retardation of the narrative, and juxtaposition make the narrative difficult to follow. On the other hand, viewers' like readers' positive affect such as amazement or happiness, and relief are elicited from the scenes or events matching their expectations. Their sympathy and empathy are manifested when they could associate themselves with the characters' situation, or when they could relate their personal experience.

Communicating interactive response refers to processing film details conveyed through interactive responses appropriate to setting and scenes rendered with visual and sound effects. Sweller's element interactivity (1988, as cited in Seel, 2008) assumes that if many elements interact, element interactivity is high; if few interact, element interactivity is low, likewise Severin's cue-summation theory (1967) claims that learning is increased as the number of available stimuli are increased. Viewers highly interact with virtually realistic scenes or events as demonstrated with their shout, reactions, and bodily movements indicating fear or amazement; or shout, clap, hug their classmates indicating relief and victory. Smith (1995, as cited in Rushton 
\& Bettison, 2010) asserted that positive emotions make the viewers continue their engagement in the film. On the other, interaction of readers with the text is cued by the narrative details in print, narrative text structure, and literary devices. As the reader interacts with the text, he may experience various emotions or reactions, and makes him more engaged in his reading.

\section{Cognitive Approach to the Three Dimensions of Visual Literacy (3D VisLit)}

The cognitive theories to viewing, processing, and responding to film provide framework for the 3D VisLit. Constructivism, schema theory, top-down processing, and interactive approach to reading; information processing, dual code theory, and cognitive load theory guide the cognitive approach to processing NLDNs. Visual cognition, cognitive theory of multimedia and film guide the cognitive approach to viewing NLDNs. Cognitive affection and theory of disequilibrium or cognitive disequilibrium guide the cognitive approach to affective and interactive responses in viewing and processing NLDNs.
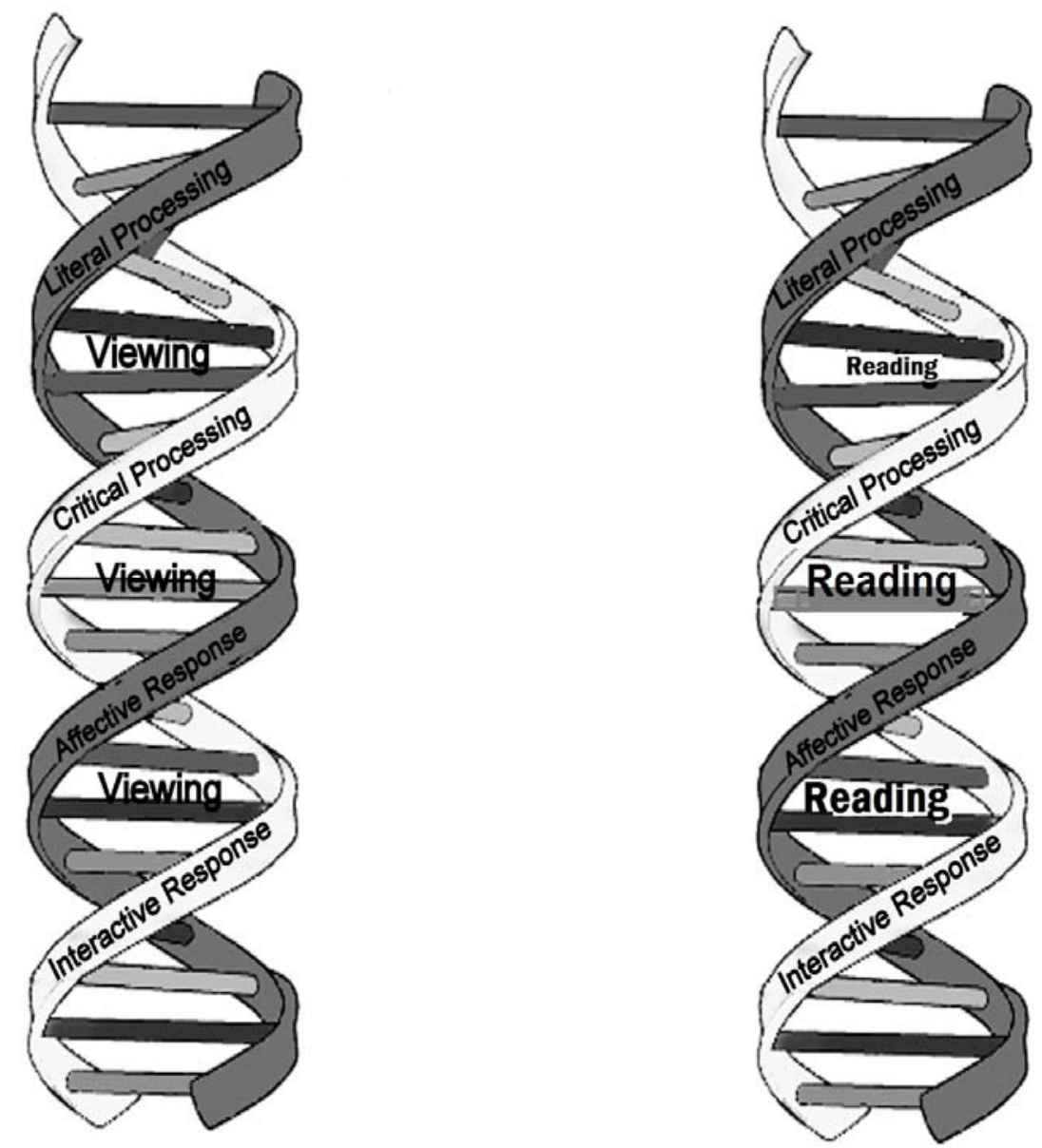

Figure 1. Three Dimensions of Visual Literacy (3D VisLit).

Bordwell (1985, as cited in Rushton \& Bettinson, 2010; as cited in Miller \& Stam, 1999), argued that spectators dynamically construct the story by responding to cues woven throughout the film, which allows the spectator to perform high level mental processes such as inference making, hypothesis framing (curiosity hypotheses and suspense hypotheses), and information processing governed by schemata which the spectator 
brings as he/she views the film. However, Currie (1995, as cited in Miller \& Stam, 1999), argued that the meaning of a film depends on how a viewer constructs it. He asserted that film possesses intrinsic meaning that the viewer should find, decode, and interpret; for if the film does not carry any intrinsic, the viewer has nothing to interpret.

Figure 1 shows the 3D VisLit. The helical model illustrates the concurrence of the three dimensions: viewing, processing, and responding. Literal and critical processing, affective and interactive responses are concurrent mental processing involved in viewing a film. When the viewer possesses, uses, finds, or discovers processing strategies in viewing a film, he uses these strategies concurrently, and responds simultaneously and spontaneously in the entire duration of viewing. Literal and critical processing strategies, affective and interactive responses are present in the strands which illustrate that they occur simultaneously, but would meet at a certain point. The three dimensions are present in the viewer's mental activity, but need to be activated to perform the necessary mental tasks influenced by the viewer's schema. Thus, when the viewer's schema is activated, the dimensions are also activated, which allows the viewer to process and respond to the film.

\section{Conclusions}

This study concluded that in determining the strategies of learners when processing NLDN, the VTTP can account for the mental processing of learners. Concurrent viewing and transcribing could account for learners' mental activities: viewing, processing, and responding that occur simultaneously. In viewing, processing, and responding to NLDN, viewers are actively involved and engaged in these simultaneous mental activities. They use literal and critical processing, processing through verbal tools and symbols, recognizing virtual reality, and communicating affective and interactive responses, which happen simultaneously.

Viewers like readers process the narrative elements: dialogue, setting, plot, character, and theme. Virtual reality effects (visual and sound) relating to the salient points of NLDNs activate and generate their critical viewing, processing, and responding to the complex narrative structure, visual and audio codes, thus, must be treated like narrative texts in print that activate schema and enhance higher-order thinking and processing skills.

Viewing involves recognizing and processing the visual codes, as well as audio codes, but is selective and critical which may be dependent on the learners' schema and information processing strategies, thus, considered critical viewing. Viewers like readers use their available processing strategies, find and discover new strategies, and integrate them to continue with their critical viewing or critical reading. They utilize their narrative, cultural and reality of the world schemata and map them with the narrative details being processed. Any mismatch makes them find and discover new strategies, combining them to proceed with their critical viewing and processing. They demonstrate, manifest, or express both affective and interactive responses appropriate to the scenes or events associated to their analytical processing.

In light of the conclusions drawn, it is therefore recommended that literacy specialists and literature teachers consider films as narrative text to enhance learners' critical viewing, processing, and response; evaluate and use viewing materials that significantly enhance learners' critical viewing, processing, and response; consider viewing as critical thinking and processing activity, critically and aesthetically interactive activity; use transcription of thought protocols in assessing the learners' critical processing of films, other viewing materials, 
and reading texts. Because of the dearth of studies on films centered on learners' processing strategies employed in viewing films, more research must be conducted to explore the cognitive processing of the 21st century learners who have increasingly immersed in digital technology.

\section{References}

Axelrod, L., \& Hone, K. (2006). Multimodal expressions: E-motional and non-verbal behaviour. Digital multimedia perception and design. London, United Kingdom: Idea Group Publishing.

Bartlett, F. C. (1932). Remembering: A study in experimental and social psychology. Cambridge, UK: Cambridge University Press.

Bordwell, D. (1985). Narration in the fiction film. Madison: University of Wisconsin Press.

Carbon, C. C., \& Albrecht, S. (2012). Bartlett's schema theory: The unreplicated "portrait d'homme” series from 1932. The Quarterly Journal of Experimental Psychology, 65(11), 2258-2270.

Craig, S. D., Driscoll, D., \& Gholson, B. (2004). Constructing knowledge from dialog in an intelligent tutoring system: Interactive learning, vicarious learning, and pedagogical agents. J. Educ. Multimedia Hypermedia, 13, 163-183.

Dancyger, K. (2011). The technique of film \& video editing: History, theory, and practice. Burlington, USA: Focal Press.

Ericsson, K. A., \& Simon, H. A. (1980). Verbal reports as data. Psychol. Rev., 87, 215-251.

Ericsson, K. A., \& Simon, H. A. (1984). Protocol analysis: Verbal reports as data. Cambridge, MA: MIT Press.

Ericsson, K. A., \& Simon, H. A. (1993). Protocol analysis: Verbal reports as data (Rev. ed.). Cambridge, MA: MIT Press.

Kuperberg, M. (2002). A guide to computer animation for TV, games, multimedia and web. Burlington, USA: Focal Press.

Lee, H. W., Lim, K. Y., \& Grabowski, B. L. (2008). Generative learning: Principles and implications for making meaning. Handbook of research on educational communications and technology. Hillsdale, New Jersey: Lawrence Erlbaum Associates.

Leedy, P. D. \& Ormrod, J. E. (2001). Practical research: Planning and design (7th ed.). Upper Saddle River, New Jersey: Prentice Hall, Inc..

Lohr, L. L., \& Gall, J. E. (2008). Representation strategies. Handbook of research on educational communications and technology. Hillsdale, New Jersey: Lawrence Erlbaum Associates.

Mandler, J. M. (1984). Stories, scripts, and scenes: Aspects of schema theory. Hillsdale, New Jersey: Erlbaum.

Mast, G., Cohen, M., \& Braudy, L. (Eds.). (1992). Film theory and critics: Introductory readings (4th ed.). New York and Oxford: Oxford University Press.

Mayer, R. E. (2001). Multimedia learning. New York: Cambridge University Press.

Mayer, R. E. (2005a). Principles for managing essential cognitive processing in multimedialearning: Segmenting, pretraining, and modality principles. In R. E. Mayer (Ed.), Cambridge handbook of multimedia learning. New York: Cambridge University Press.

Mayer, R. E. (2005b). Principles for reducing extraneous processing in multimedia learning: Coherence, signalling, redundancy, spatial contiguity, and temporal contiguity principles. In R. E. Mayer (Ed.), Cambridge handbook of multimedia learning. New York: Cambridge University Press.

Mayer, R. E., \& Gallini, J. K. (1990). When is an illustration worth ten thousand words?. Journal of Educational Psychology, 82(4), 715-726.

McKay, S. L. (1993). Agendas for second language literacy. New York: Cambridge University Press.

Miller, T., \& Stam, R. (Eds.). (1999). A companion to film theory. Malden, Massachusetts: Blackwell Publishers.

Ohanian, T. A., \& Phillips, M. E. (1996). Digital filmmaking: The changing art and craft of making motion pictures. Burlington, USA: Focal Press.

Piaget, J. (1952). The origins of intelligence in children. New York: International Universities Press.

Plutchik, R., \& Conte, H. R. (1997). Circumplex models of personality and emotions. Washington, DC: American Psychological Association.

Rosenblatt, L. (1991). Literary theory. In J. Flood, J. Jensen, D. Lapp, \& J. Squire (Eds.), Handbook of research on teaching the English language arts (pp. 57-62). New York: Macmillan.

Rumelhart, D. E. (1980). Schemata: The building blocks of cognition. Theoretical issues in reading comprehension. Hillsdale: Lawrence Erlbaum Associates.

Rumelhart, D. E., McClelland, J. L., \& The PDP Research Group. (1986). Parallel distributed processing: Explorations in the microstructure of cognition. Cambridge: M.I.T. Press.

Rushton, R., \& Bettinson, G. (2010). What is film theory? An introduction to contemporary debates. Berkshire, England: Open University Press. 
Salovey, P., \& Mayer, J. D. (1990). Emotional intelligence. Imagination, Cognition and Personality, 9, 185-211.

Seel, N. (2008). Empirical perspectives on memory and motivation. Handbook of research on educational communications and technology. Hillsdale, New Jersey: Lawrence Erlbaum Associates.

Severin, W. J. (1967). Cue summation in multiple channel communication (Unpublished doctoral dissertation). University of Wisconsin, Madison.

Singer, J. L., \& Singer, D. G. (1983). Implications of childhood television viewing for cognition, imagination, and emotion. In J. Bryant \& D. R. Anderson (Eds.), Children's understanding of television: Research on attention and comprehension (pp. 265-295). New York: Academic Press.

Stanovich, K. E. (1980). Toward an interactive-compensatory model of individual differences in the development of reading fluency. Reading Research Quarterly, 16, 32-71.

Sweller, J. (1988). Cognitive load during problem solving: Effects on learning. Handbook of research on educational communications and technology. Hillsdale, New Jersey: Lawrence Erlbaum Associates.

van Gog, T., \& Paas, F. (2008). Data collection and analysis. Handbook of research on educational communications and technology. Hillsdale, New Jersey: Lawrence Erlbaum Associates.

Vygotsky, L. S. (1978). Mind in society: The development of higher psychological processes. Cambridge: Harvard University Press. 